\title{
Complications associated with central venous lines for paediatric oncology patients at Universitas Academic Hospital, Bloemfontein, from 1992 to 2018
}

\author{
L Mokone $^{1}$; P Ndove ${ }^{1}$; K Magooa ${ }^{1}$; K Tsilo ${ }^{1}$; R Rampeta ${ }^{1}$; E Brits, ${ }^{2}$ MMed (Paed Surg), FC Paed Surg; G Joubert, ${ }^{3}$ MSc \\ ${ }^{1}$ Faculty of Health Sciences, University of the Free State, Bloemfontein, South Africa \\ ${ }^{2}$ Department of Surgery, School of Clinical Medicine, University of the Free State, Bloemfontein, South Africa \\ ${ }^{3}$ Department of Biostatistics, School of Biomedical Sciences, University of the Free State, Bloemfontein, South Africa
}

Corresponding author: E Brits (BritsE@ufs.ac.za)

\begin{abstract}
Background. Central venous access devices are associated with complications such as central-line infections and systemic sepsis. Objective. To determine the complication rates associated with central venous lines used to administer chemotherapy at the Paediatric Haematology Oncology Unit, Universitas Academic Hospital, Bloemfontein, from January 1992 to March 2018.

Methods. A retrospective descriptive analysis of paediatric oncology patients who received intravenous catheterisation and were treated at the unit. Cases with incomplete data, age $>16$ years and treatment after 31 March 2018 were excluded.

Results. Records of 293 Hickman lines were analysed. The median patient age was 64.7 months. Sepsis was noted in $13.3 \%$ of the cases; no in situ local complications were found in $62.5 \%$ of the lines. Of the 39 cases that presented with line sepsis, $23.1 \%$ showed no signs of systemic sepsis, whereas $61.5 \%$ were neutropenic and septic. In total, 190 patients had symptoms of systemic sepsis and accompanying neutropenia. Of these, $67.4 \%$ did not develop in situ line complications. Lines were removed mostly because the end of treatment had been reached (44.7\%); line sepsis was noted as the reason for removal in only $16.3 \%$ of cases.

Conclusion. Despite the study population having a high risk for infections because of a young age, a large proportion of haematological malignancies and surgical placement of Hickman lines, there was a low incidence of line sepsis complications. The special care taken intraoperatively and meticulous aseptic postoperative handling and maintenance are suggested as contributing to limited infective complications.
\end{abstract}

S Afr J Child Health 2021;15(4):189-192. https://doi.org/10.7196/SAJCH.2021.v15i4.1778

A venous access device is a catheter designed for continuous access to the venous system, which may be required for long-term parenteral feeding, administration of intravenous (IV) fluids and medication, or taking blood samples. ${ }^{[1,2]}$ Several types of central venous access devices (CVADs) are used, such as external tunnelled cuffed catheters (e.g. Hickman and Broviac lines), chemo ports (totally implanted catheter ports) and peripherally inserted central catheters (PICCs).

In oncology patients, CVADs are typically used for administering pro-inflammatory chemotherapy agents, bone marrow transplants, antibiotics and fluids, and for blood sampling. With these patients needing medium- to long-term treatment ( 2 - 3 years), ${ }^{[3]}$ CVADs are commonly used to avoid multiple needle pricks of peripheral veins, thereby decreasing their anxiety and improving patients' quality of life during the treatment period, especially in patients with difficult peripheral IV access (non-visible or non-palpable veins). ${ }^{[4-6]}$

Central venous catheters may cause complications in up to $40 \%$ of paediatric patients. ${ }^{[7]}$ Common complications seen in paediatric patients with chemo ports are those associated with bloodstream infections, local skin infections, wound dehiscence, mechanical complications, venous thrombosis and skin necrosis. ${ }^{[8]}$ The use of CVADs can also be associated with arterial puncture, haemothorax, stroke, arrhythmias and nerve damage. ${ }^{[9]}$

Although fewer complications and risks are associated with Hickman lines, just like with any other CVAD, their lumens may become blocked, they may become infected, and the cut-down may result in scarring of the skin. ${ }^{[2]}$ Precaution must be taken not to contaminate the exposed external part of the line. External tunnelled cuffed catheters are more prone to line sepsis than chemo ports (4.7 v. 1.5 episodes per 1000 catheter days). ${ }^{[10]}$

PICC lines are often used in oncology patients receiving shortterm treatment or in patients outside the oncological setting, but can be associated with complications such as extravasation $(81.6 \%)$ and infections (78.3\%). ${ }^{[1]}$ Multiple attempts to insert a PICC line may introduce infection, which is a risk in an already immunocompromised patient. In addition, the use of PICCs can lead to compromised integrity of the peripheral veins over time, which makes reliable peripheral venous access increasingly difficult in these patients. Other options of CVADs which are more reliable and less prone to complications, are therefore preferred. ${ }^{[11]}$

Chemo ports are totally implanted and therefore do not have an external part that can become contaminated and infected by direct contact to the environment. The only maintenance required is monthly flushing of the line when not in use. Patients with a chemo port can bath, shower and swim as normal. Because of these benefits, and also a lower rate of removal due to mechanical complications, chemo ports are preferred over Hickman lines for central venous access. ${ }^{[1]}$

Risk factors for CVAD complications include the type of CVAD, underlying disease (more common in haematological malignancies) and patient age (more common in younger patients). ${ }^{[7]}$ Neutropenia is one of the most important risk factors for the outbreak of infections. ${ }^{[12-15]}$ 
The choice of a CVAD depends on the typical complication rate of the approach and the risk of thrombotic or septic complications associated with a specific device, together with the planned therapy course and the clinical experience of the provider. ${ }^{[16,17]}$ Choosing the appropriate device for the oncology patient should be part of proactive vascular access planning.

The Paediatric Haematology Oncology Unit at the Universitas Academic Hospital, Bloemfontein, mainly uses Hickman lines. This is due to availability and most of the staff at the hospital being familiar with and skilled in using this type of CVAD. The preferred site of insertion is the right internal jugular vein, but the left internal jugular vein is also used if thrombosis has occurred on the right side following a previous line.

These lines are inserted under general anaesthesia in theatre by paediatric surgeons. The device is placed in an iodine solution prior to insertion, provided that the patient is not allergic to iodine. Cefazolin is given as a prophylactic antibiotic at induction of anaesthesia. The device can be inserted either through ultrasound-guided percutaneous puncture (Seldinger technique) or a surgical cut-down using anatomical landmarks. To confirm correct positioning of the catheter tip, fluoroscopy is performed intraoperatively.

The open cut-down technique in the neck is commonly used at Universitas Academic Hospital, as the Hickman lines available at the facility do not always support the Seldinger technique because of instrumentation constraints. However, if the equipment supports the use of the Seldinger technique, it is preferred. The line is tunnelled to exit just inferior to the xiphisternum. This exit position supports better postoperative maintenance of the line (e.g. enough space for dressing application to protect the line against environmental contamination). The tip of the line is positioned in the superior vena cava (SVC).

The line is then flushed with heparinised saline and the external part is covered with a sterile dressing on the chest and upper abdomen wall. In patients who had a previous line, Doppler ultrasound is performed before placement of the new device to evaluate the vessel for patency and possible thrombus formation. Routine evaluation for vessel thrombosis is performed only if the patient presents with symptoms and signs of vessel occlusion (SVC syndrome, swollen limbs).

The combined Paediatric Haematology Oncology and Paediatric Surgery Unit at Universitas Academic Hospital is the only referral centre for children with malignancies and index cases of paediatric surgical conditions in central South Africa. This unit serves the Free State, Northern Cape and selected areas of the North West and Eastern Cape provinces. It also serves as a referral unit for Lesotho.

According to the protocol used in our unit, the catheter may only be used 48 hours after placement. The external part of the line is cleaned with chlorhexidine soap and flushed with heparinised saline twice per week, regardless of whether the patient is admitted or at home. Likewise, IV sets are replaced with new ones and the external part of the line is dressed with a clean dressing against the patient's chest and upper abdomen wall twice per week. ${ }^{[18]}$

Catheter-associated bloodstream infection (CABSI) is diagnosed by a positive blood culture taken from the line when the patient is clinically septic (i.e. presenting with fever) and is treated with antibiotics administered through the line. Initial treatment involves piperacillin/tazobactan and amikacin used empirically, but this can be changed according to sensitivity results of the blood culture. If the same organism is cultured after a week of treatment, the line is regarded septic and consequently removed. Blood samples for culture are never taken from a peripheral site while the Hickman line is functional. Sepsis at the tunnel exit site and pocket is treated conservatively by draining an abscess, wound cleaning and administering antibiotics without removal of the line. If there is no improvement, the line is removed and the tip is sent for culture. ${ }^{[15]}$ Line cultures are performed only per indication when a patient appears septic, not as routine. Blood sampling is performed as a sterile procedure and any growth is regarded as pathogenic.

This study assessed the complications of central venous lines for chemotherapy in paediatric oncology patients treated in the Paediatric Haematology Oncology Unit at the Universitas Academic Hospital in Bloemfontein from January 1992 to 31 March 2018.

Further objectives were to describe subgroups that developed line sepsis complications and had increased risk factors for line sepsis.

\section{Methods}

This was a retrospective descriptive study of paediatric oncology patients (16 years or younger) who received CVADs and were treated in the Paediatric Haematology Oncology Unit at Universitas Academic Hospital in Bloemfontein from January 1992 to March 2018.

A pilot study was run using data from three cases.

\section{Data collection}

Data were collected from the unit's database and included: the patient's age (months) at line insertion, diagnosis and reason for line insertion; data on specific insertion parameters (site of insertion, days in situ, any complications at insertion); and post-insertion developments (namely purpose served without complications during treatment, in situ line complications, reason for line removal, and presence of sepsis). Data collection and preparation for analysis were shared by the authors. All authors verified the information recorded.

\section{Data analysis}

As no changes were made to the methodology after the pilot study, those three cases could be included in the final dataset. Data were entered into a spreadsheet (MS Excel) for statistical analysis. Categorical variables were summarised as frequencies and percentages, whereas means, standard deviations and percentiles are reported for numerical variables.

\section{Ethical considerations}

Approval for the study was obtained from the Health Sciences Research Ethics Committee of the University of the Free State (ref. no. UFS-HSD2018/0389/3010) and the Free State Department of Health. To ensure patient confidentiality, no identifying information such as names or admission numbers was captured.

\section{Results}

\section{Clinical characteristics $(\boldsymbol{N}=\mathbf{2 9 3})$}

A total of 300 Hickman lines were inserted over the study period; seven cases were excluded owing to missing information or the patient being older than 16 years. The final sample size was therefore 293 lines. The median patient age was 64.7 months (range: 3.2 - 193.3 months). The median days in situ were 132 (range: 0 - 769 days). The most common patient diagnoses were leukaemia (60.8\%), solid tumours (28.3\%) and lymphoma (10.9\%). Insertion sites were recorded as the internal jugular vein $(64.2 \%)$ or subclavian vein (1.7\%); no insertion site was recorded in $34 \%$ of the cases. The main reasons for line insertion included: protocol requirement (56.0\%); difficult peripheral IV access (23.2\%); and patient requests (16.7\%). Diverse other reasons accounted for the remainder of cases.

Complications associated with line placement (procedural complications) were found in only $12 \%$ of cases, and were recorded 
as: bleeding (3.4\%), incorrect position (3.1\%), failure to insert (1.0\%), pneumothorax $(0.7 \%)$, and 'other' (3.8\%). In situ complications were encountered in $37.5 \%$ of the cases, of which the most common complications included line sepsis (13.3\%), blockage (6.1\%) and the line having pulled out (5.5\%) (Table 1).

Complications (procedural and in situ) were recorded in less than half $(43.3 \%)$ the cases. CABSI was noted as reason for line removal in $14.3 \%$ of cases. In the remaining cases, removal was due to the end of treatment (37.9\%), relapse (5.1\%), line blockage (10.6\%) or diverse 'other' reasons (21.2\%). In $10.9 \%$ of cases, patients died with their lines in situ. Overall, $75.1 \%$ of the lines served their purpose.

\section{In situ complications of line sepsis $(\boldsymbol{n}=39)$}

Line sepsis was an in situ complication in $13.3 \%$ of the total number of cases (Table 1). No systemic sepsis was noted in almost a quarter of these 39 cases $(23.1 \%)$, whereas $61.5 \%$ of this set of cases were both neutropenic and septic, and $15.4 \%$ presented with symptoms of sepsis without neutropenia. The diagnoses in these patients were leukaemia (56.4\%), solid tumours (35.9\%) and lymphoma (7.7\%).

Line sepsis as a reason for removal was recorded in $38.5 \%$ of these cases (Fig. 1). The total number of line days over the course of the study period was 39889 . The number of sepsis episodes $(n=39)$ translated to a total rate of 0.98 episodes per 1000 catheter days. The median number of line days for cases complicated with sepsis was 143 days (range: 14 - 704 days). The lines still served their purpose in $79.5 \%$ of cases.

\section{Symptoms of systemic sepsis and accompanying neutropenia $(n=190)$}

Symptoms of systemic sepsis and accompanying neutropenia were noted in 190 cases (64.8\%). Of these 190 lines, 12.6\% developed CABSIs, with blockage recorded as the second most common in situ line complication (5.8\%). Approximately two-thirds (67.4\%) of patients did not develop any in situ line complications. The diagnoses in the patients in this subgroup were leukaemia (71.6\%), solid tumours (17.3\%) and lymphoma (11.0\%).

Table 1. In situ local complications $(N=293)$

\begin{tabular}{ll}
\hline Complications & $\boldsymbol{n}(\%)$ \\
\hline None & $183(62.5)$ \\
Sepsis & $39(13.3)$ \\
Pulled out & $16(5.5)$ \\
Blocked & $18(6.1)$ \\
Ruptured & $3(1.0)$ \\
Unable to take blood & $5(1.7)$ \\
Swelling & $10(3.4)$ \\
Other & $19(6.5)$
\end{tabular}

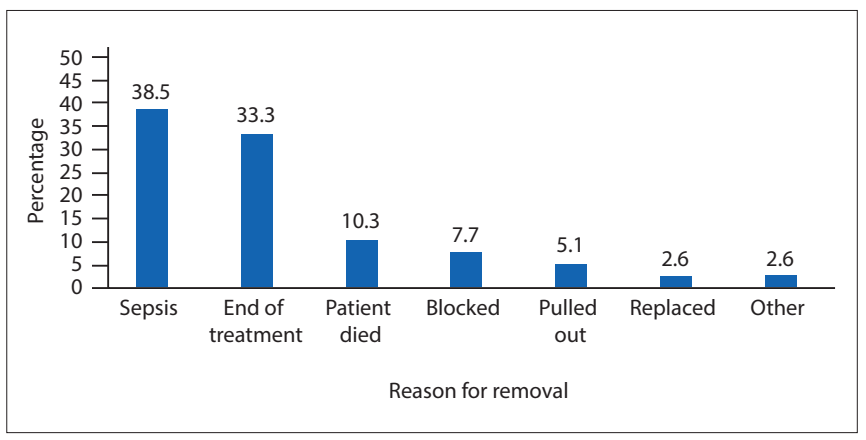

Fig. 1. Reasons for line removal in cases with sepsis $(\mathrm{n}=39)$
Only $16.3 \%$ of the 190 lines were removed because of sepsis. We found that $14.7 \%$ of patients in this group died with their lines in situ and $44.7 \%$ had their lines removed because they reached the end of their treatment.

The median number of catheter days for cases with systemic sepsis and accompanying neutropenia was 152 (range: 7 - 769 days). The majority of these lines $(n=161 / 190 ; 84.7 \%)$ still served their purpose.

\section{Discussion}

The median age of 64.7 months (5.4 years) at line placement is younger than the 7.1 years reported by Adler et al. ${ }^{[10]}$ who noted a young age being a risk factor for complications developing with the use of CVADs. ${ }^{[9]}$ Patients with leukaemia made up $60.8 \%$ of our cohort and lymphoma accounted for $10.9 \%$, meaning haematological malignancy featured in almost three-quarters $(71.7 \%)$ of the patients in our study. This is a higher proportion than reported by Adler et al. ${ }^{[10]}(57.4 \%)$. Haematological malignancies and neutropenia are risk factors for line sepsis. ${ }^{[12-15]}$ Data in the current study showed 0.98 sepsis episodes per 1000 catheter days, which is lower than the rates reported by Adler et al.$^{[10]}$ (4.7 episodes per 1000 catheter days) and Basford et al. ${ }^{[19]}$ (11.4 sepsis episodes per 1000 catheter days). The median of 132 in situ line days is comparable with the 140.7 days reported by Adler et al. ${ }^{[10]}$

Procedural complications were noted in $12.0 \%$ of cases, with in situ complications in $37.5 \%$ of cases. Some overlap of complications resulted in a combined complication figure of $43.3 \%$. Almost all the lines were inserted surgically, as per the preferred method during the period reviewed in this study. It is well described that surgically placed lines are associated with more infectious and mechanical complications ${ }^{[19]}$ compared with radiologically assisted (i.e. ultrasound-guided) percutaneous techniques in the paediatric oncological setting. However, the combined complication rate (43.3\%) in our study compares well with the rate of $59.6 \%$ (infectious or mechanical complications) reported by Basford et al. ${ }^{[19]}$ for external tunnelled catheters.

The observed rate of line sepsis (CABSI) in our study (13.3\%) compares favourably with that reported for chemo ports in other studies $\left(21.7 \%{ }^{[19]} 23.5 \%,{ }^{[10]}\right)$ and is better than rates reported for external tunnelled catheters $\left(36.5 \%,{ }^{[19]} 35.4 \%,{ }^{[10]}\right.$ and $\left.47.1 \%{ }^{[20]}\right)$.

In the cases that presented with line sepsis, $61.5 \%$ had a combination of neutropenia and systemic sepsis. Of these, almost two-thirds (64.1\%) were diagnosed with haematological malignancies. Lines were removed owing to sepsis in only $38.5 \%$ of these cases and therefore CVAD use could be successfully salvaged by the administration of antibiotics in a large number of cases.

In the $64.8 \%$ of the study population that had neutropenia and symptoms of systemic sepsis, $67.4 \%$ had no in situ line complications and only $12.6 \%$ developed line sepsis, despite being neutropenic and systemically ill, both risk factors for line sepsis. Lines were removed owing to sepsis in a smaller proportion (16.3\%) than expected. Almost half of these patients reached the end of their treatment and the lines would have been removed in any case. Central lines served their purpose in a high proportion of the cohort (84.7\%).

\section{Study limitations}

The retrospective nature of the study is regarded as a limitation, as vital information was missing from some cases, which resulted in their being excluded from the analysis.

\section{Conclusion}

Our analysis shows a low percentage of line sepsis (CABSI) in this cohort (13.3\%), despite the study population being young, a large 
proportion of haematological malignancies being included and the use of Hickman lines placed surgically. With these characteristics, our study population would have had a high risk for infective complications. The special care taken intraoperatively and the meticulous aseptic handling and maintenance of these lines postoperatively (by teaching staff to manage the lines within strict protocols) may have contributed to the low infective complication rates.

Further studies are recommended to investigate the role of neutropenia and haematological malignancies (leukaemia and lymphoma) in the development of line sepsis.

\section{Declaration. None.}

Acknowledgements. The authors wish to thank Prof. DK Stones and Dr Z Henning for initiating the study, Dr SM le Grange for her contributions and the University of the Free State for the opportunity to conduct the study. We also thank Ms T Mulder for technical preparation of the manuscript. Author contributions. LM, PN, KM, KT and RR developed the study protocol, collected the data and prepared the initial draft of the manuscript. EB was the study supervisor, suggested the concept and assisted with protocol development, data collection and interpretation, and manuscript preparation. GJ assisted with study conceptualisation, analysed the data and assisted with interpretation and manuscript development.

Funding. None.

Conflicts of interest. None.

1. Mosby's Medical Dictionary. Venous access device. 9th ed. St Louis, MO: Elsevier, 2009.

2. Guy's and St Thomas' NHS Foundation Trust. Having a Hickman-line insertion. 2017. https://pdf4pro.com/amp/cdn/hickman-line-insertion-in-theguy-s-and-st-thomas-3f91e5.pdf (accessed 11 April 2018).

3. DeWitty RL, Siram SM, Balkissoon J. Vascular access in the cancer patient. J Natl Med Assoc 1986;78(4):289-291.

4. Wang SC, Tsai CH, Hou CP, et al. Dislodgement of port-A catheters in pediatric oncology patients: 11 years of experience. World J Surg Oncol 2013;11:191. https://doi.org/10.1186/1477-7819-11-191

5. Statter MB. Peripheral and central venous access. Semin Pediatr Surg 1992;1(3):181-187.

6. Cheung E, Baerlocher MO, Asch M, Myers A. Venous access: A practical review for 2009. Can Fam Physician 2009;55(5):494-496.
7. Fratino G, Molinari AC, Parodi S, Longo P, Castagnola E, Haupt R. Central venous catheter-related complications in children with oncological/ hematological diseases: An observational study of 418 devices. Ann Oncol 2005;16(4):648-654. https://doi.org/10.1093/annonc/mdi111

8. Aparna S, Ramesh S, Appaji L, et al. Complications of chemoport in children with cancer: Experience of 54,100 catheter days from a tertiary cancer center of Southern India. South Asian J Cancer 2015;4(3):143-145. https://doi. org/10.4103/2278-330X.173179

9. Kusminsky RE. Complications of central venous catheterization. J Am Coll Surg 2007;204(4):681-696. https://doi.org/10.1016/j.jamcollsurg.2007.01.039

10. Adler A, Yaniv I, Steinberg R, et al. Infectious complications of implantable ports and Hickman catheters in paediatric haematology-oncology patients. J Hosp Infect 2006;62(3):358-365. https://doi.org/10.1016/j.jhin.2005.08.019

11. Hooda B, Lalani G, Fadoo Z, Billoo G. Implantable port devices are catheters of choice for administration of chemotherapy in pediatric oncology patients - a clinical experience in Pakistan. Ann N Y Acad Sci 2008;1138:43-46. https://doi. org/10.1196/annals.1414.007

12. Biagi E, Arrigo C, Dell'Orto MG, et al. Mechanical and infective central venous catheter-related complications: A prospective non-randomized study using Hickman and Groshong catheters in children with hematological malignancies. Support Care Cancer 1997;5(3):228-233. https://doi.org/10.1007/ s005200050065

13. McLean TW, Fisher CJ, Snively BM, Chauvenet AR. Central venous lines in children with lesser risk acute lymphoblastic leukemia: Optimal type and timing of placement. J Clin Oncol 2005;23(13):3024-3029. https://doi. org/10.1200/JCO.2005.12.097

14. Nam SH, Kim DY, Kim SC, Kim IK. Complications and risk factors of infection in pediatric hemato-oncology patients with totally implantable access ports (TIAPs). Pediatr Blood Cancer 2010;54(4):546-551. https://doi.org/10.1002/ pbc. 22286

15. Funaki B. Subcutaneous chest port infection. Semin Intervent Radiol 2005;22(3):245-247. https://doi.org/10.1055/s-2005-921959

16. Ruesch S, Walder B, Tramèr RM. Complications of central venous catheters: Internal jugular versus subclavian access - systematic review. Crit Care Med 2002;30(2):454-460. https://doi.org/10.1097/00003246-200202000-00031

17. Cotogni P, Barbero C, Garrino C, et al. Peripherally inserted central catheters in non-hospitalized cancer patients: 5-year results of a prospective study. Support Care Cancer 2015;23(2):403-409. https://doi.org/10.1007/s00520-014-2387-9

18. Bishop L, Dougherty L, Bodenham A, et al. Guidelines on the insertion and management of central venous access devices in adults. Int J Lab Hemato 2007;29(4):261-278. https://doi.org/10.1111/j.1751-553X.2007.00931.x

19. Basford TJ, Poenaru D, Silva M. Comparison of delayed complications of central venous catheters placed surgically or radiologically in pediatric oncology patients. J Pediatr Surg 2003;38(5):779-788. https://doi.org/10.1016/ jpsu.2003.50168

20. Coady K, Ali M, Sidloff D, Kenningham RR, Ahmed S. A comparison of infections and complications in central venous catheters in adults with solid tumours. J Vasc Access 2015;16(1):38-41. https://doi.org/10.5301/jva.5000300

Accepted 23 February 2021 